\title{
Folate receptor-mediated targeted polymeric gadolinium complexes for magnetic resonance imaging in pulmonary tumor xenografts
}

\author{
ZHENG YUAN $^{1,2^{*}}$, WEN-TAO LI ${ }^{1 *}$, XIAO-DAN YE ${ }^{3}$, SHI-YUAN LIU ${ }^{4}$ and XIANG-SHENG XIAO ${ }^{4}$ \\ ${ }^{1}$ Department of Radiology, Fudan University Shanghai Cancer Center, Shanghai 200032; ${ }^{2}$ Department of Radiology, \\ Nanjing Jinling Hospital, Clinical School of Medical College, Nanjing University, Nanjing, Jiangsu 210002; \\ ${ }^{3}$ Department of Radiology, Shanghai Chest Hospital Affiliated to Shanghai Jiaotong University, \\ Shanghai 200030; ${ }^{4}$ Department of Radiology, Affiliated Changzheng Hospital, \\ The Second Military Medical University, Shanghai 200003, P.R. China
}

Received December 14, 2011; Accepted February 6, 2012

DOI: $10.3892 / \mathrm{etm} .2012 .504$

\begin{abstract}
Targeted delivery is a highly desirable strategy for diagnostic imaging due to enhanced efficacy and reduced dosage/toxicity. The need to develop target-specific magnetic resonance imaging (MRI) contrast agents to aid in disease characterization is highly essential. In this study, a specific contrast agent, Gd-DTPA-poly-L-lysine (PL-Gd-DTPA)folate, was synthesized and evaluated for its efficacy as a targeted agent for the imaging of tumors that overexpress the folate receptor. Folic acid was conjugated to PL-Gd-DTPA via the $\varepsilon$-amino groups. The receptor binding properties of folate-PL-Gd-DTPA were studied in cultured tumor cells that overexpressed the folate receptor. The tumor-selecting properties of folate-PL-Gd-DTPA were then evaluated in BALB/c mice bearing subcutaneously implanted folate receptor-positive tumors. Tissue MR signal intensities were measured at six different time-points. In the in vitro study, the folate-PL-Gd-DTPA was able to bind to these cells, which overexpressed the folate receptor, as with free folic acid. Excellent tumor selectivity was also shown in the animal model; after the success of injection of folate-PL-Gd-DTPA, a maximum intensity increase of $125.4 \%$ was observed from pre-injection compared to post-injection images of the tumor at the $48 \mathrm{~h}$ time-point. The liver enhancement was non-specific
\end{abstract}

Correspondence to: Dr Xiao-Dan Ye, Department of Radiology, Shanghai Chest Hospital Affiliated to Shanghai Jiaotong University, 241 West Huai Hai Road, Shanghai 200030, P.R. China

E-mail: yuanyxd@163.com

Dr Shi-Yuan Liu, Department of Radiology, Affiliated Changzheng Hospital, The Second Military Medical University, 415 Feng Yang Road, Shanghai 200003, P.R. China

E-mail: liushiyuan@vip.163.com

*Contributed equally

Key words: folate receptor, pulmonary tumor, targeted contrast agents, magnetic resonance imaging, gadolinium-DTPA and the muscle signal intensity at any time-point after injection showed no statistical difference with that observed before injection. Folate-PL-Gd-DTPA is a promising, novel receptorspecific MRI contrast agent with potential applications in the imaging of human folate receptor-positive tumors.

\section{Introduction}

Targeted delivery of contrast agents is a highly desirable strategy for enhancing efficacy and reducing unintended side effects and toxicity (1-3). Low et al also stated the need for more sensitive and accurate tumor markers as well as imaging studies (4). The development of new and improved tumor selective magnetic resonance imaging (MRI) contrast agents is clinically desirable as a means of: i) detecting and/or confirming the presence and location of primary and metastatic lesions; ii) probing biochemical features of neoplastic tissue that have implications for tumor staging and/or treatment planning; and iii) monitoring tumor response to treatment (5).

Macromolecular contrast agents (>20,000 Da), due to their larger size, enable longer imaging times and possess higher relaxation rates resulting from their longer rotational correlation times (6). These agents have been used to enhance tumors via active targeting. Active targeting utilizes a specific cell surface molecule that is either unique or overexpressed in the tissue of interest. This type of targeting is more challenging due to large amounts of magnetic label in the tissue of interest required in order to achieve a sufficient diagnostic signal. Unger et al used an anti-carcinoembryonic antigen (CEA) antibody conjugated to $\sim 1.5 \mathrm{Gd}$ atoms/antibody to enhance human colon carcinoma implanted in the thighs of hamsters (7). The use of antibodies as target-specific molecules experience problems that include rapid clearance by the reticuloendothelial system, decreased affinity of the antibody following conjugation of the magnetic label, circulating free antigen, insufficient tumor penetration, binding of antibodies to non-specific Fc receptors, and possible changes in the antigen over time (7-9).

To avoid these problems, targeting endogenous transport mechanisms within cells have been suggested, one of which is the recycling high-affinity folate receptor (hFR) pathway 
(10-12). The folate receptor is a glycosylphosphatidylinositolanchored, high-affinity, folate-binding protein overexpressed in various types of human tumors (13-17), where it functions by capturing folate to feed rapidly dividing tumor cells (17-19). Two folate receptor isoforms have been identified in human cell membrane, $\alpha$ and $\beta$. The type $\alpha$ receptor is frequently overexpressed in epithelial-lineaged tumors and type $\beta$ in nonepithelial-derived tumors. Both isoforms show a high affinity for folic acid.

There has been much interest in exploiting the folate receptor for tumor-specific-targeted delivery of imaging or therapeutic agents. Earlier efforts in targeting the folate receptor were focused on the use of conjugates of antibodies against the folate receptor. An alternative strategy has been developed for folate receptor targeting in which the ligand, folic acid, is covalently linked to the molecule to be delivered. Folic acid (molecular weight 441.4) is a high-affinity ligand for the folate receptor $\left(\mathrm{kD} \sim 10^{-10} \mathrm{~mol} / \mathrm{l}\right)$. Folate conjugates are taken into cultured tumor cells by binding to the folate receptor on the cellular surface followed by receptor-mediated endocytosis. This targeting strategy has been exploited in the receptor-mediated delivery of proteins, liposomes, gene transfer vectors, chemotherapeutic agents and radioimaging agents into tumor cells. By contrast, non-proliferating normal cells are severely restricted in possessing folate receptors $(17,20)$. Thus, folate receptors provide highly selective sites that differentiate tumor cells from normal cells. Folate conjugates are also suitable for targeted delivery because of folate's lack of immunotoxicity and its role in receptor-mediated endocytosis.

In this study, a folate receptor-targeted, polymeric gadolinium complex was utilized, where the $\varepsilon$-amino groups of poly-L-lysine were conjugated to $~ 56$ Gd-DTPA molecules per folic acid to increase the number of gadolinium (21). Using this folate conjugate, we successfully produced the contrastenhanced, folate receptor-targeted tumor image in vivo in pulmonary tumor xenografts.

\section{Materials and methods}

Contrast agent. All reagents used in the synthesis were commercial products. Diethylenetriaminepentaacetic acid (DTPA) (10 g) was added to $30 \mathrm{ml}$ acetic anhydride and $15 \mathrm{ml}$ pyridine and stirred for $6 \mathrm{~h}$ at $60^{\circ} \mathrm{C}$. CaDTPA in white solid form was obtained after cooling and drying. Polylysine (10 mg) was dissolved in $3 \mathrm{ml}$ sodium carbonate buffer (200 mM NaHCO $3 / \mathrm{Na}_{2} \mathrm{CO}_{3}$, pH 9.6), to which CaDTPA solution (polylysine:CaDTPA 1:86 in molar ratio) was added. PL-DTPA resulted after stirring the mixture solution for $16 \mathrm{~h}$ in an icebath. PL-Gd-DTPA was synthesized by adding $\mathrm{GdCl}_{3}$ solution to PL-DTPA solution $\left(\mathrm{Gd}^{3+}\right.$ :DTPA in equal ratio) and stirring for 24-30 h at room temperature. Folic acid in $100 \mathrm{mM}$ $\mathrm{NaHCO}_{3} / \mathrm{Na}_{2} \mathrm{CO}_{3}$ buffer, $\mathrm{pH}$ 9.6, was conjugated to PL-GdDTPA through ECD linkage (folic acid:PL-Gd-DTPA:ECD 5:1:5.3 in molar ratio). The free folic acid was separated by gel filtration [Sephadex CL-4B column $(1 \times 25 \mathrm{~cm})]$. The complex of folic acid and PL-Gd-DTPA was collected and iceconcentrated, and used for animal imaging experiment after aseptic filtration. ICP-AES and HPLC were used for the $\mathrm{Gd}^{3+}$ concentration quantification (21).
Cell culture and in vitro specificity studies. The H460 human lung adenocarcinoma cells, expressing the hFR were obtained from the Shanghai Cancer Institute (Shanghai, China). Cell lines were cultured continuously as a monolayer at $37^{\circ} \mathrm{C}$ in a humidified atmosphere containing $5 \% \mathrm{CO}_{2}$ in folate-free RPMI-1640 medium supplemented with $10 \%$ fetal bovine serum, $50 \mathrm{U} / \mathrm{ml}$ penicillin and $50 \mu \mathrm{g} / \mathrm{ml}$ streptomycin. The final folate concentration (with the fetal bovine serum as the only source of folate) falls in the range of the physiological concentration in human serum. Because of their high folate content $(2 \mu \mathrm{mol} / \mathrm{l})$, which leads to the downregulation and presaturation of the cellular folate receptor, regular culture media were not used.

The cultured cells were washed with $1 \mathrm{ml}, 0.01 \mathrm{M}, \mathrm{pH} 7.4$, phosphate-buffered saline. Following $3 \mathrm{~min}$ of centrifugation at 2,000 rpm, cells were aliquoted into tubes consisting of $1 \times 10^{6}$ cells $/ 0.5 \mathrm{ml}$. Folic acid labeled ${ }^{125} \mathrm{I}\left(\sim 5 \times 10^{6}\right)$ was added to each tube. Then, for the competition inhibition studies, folic acid or folate-PL-Gd-DTPA $100 \mu \mathrm{l}$ was added to each tube in different concentrations, stored at $4^{\circ} \mathrm{C}$ overnight. Cells were then washed with $2 \mathrm{ml}, 0.01 \mathrm{M}$, pH 7.4, phosphate-buffered saline, following $15 \mathrm{~min}$ of centrifugation at 3,000 rpm. After blotting supernatant, radioactivity was measured using the $\gamma$-ray emissive counter. Log-curves were drawn between $\mathrm{B} / \mathrm{B}_{0}$ and different concentrations of folic acid or folate-PL-Gd-DTPA.

Animal model. Pulmonary tumors were induced in BALB/c mice 5-6 weeks of age (Shanghai Cancer Institute, Shanghai, China) by subcutaneously injecting $10^{7}-10^{8}$ human lung adenocarcinoma $\mathrm{H} 460$ cells expressing the hFR in the subscapular area on the dorsal surface. Mice were housed in polycarbonate microisolator cages, and tumor sizes were monitored three times per week. Tumors reaching a diameter of $\sim 1 \mathrm{~cm}$ after 12-14 weeks of inoculation were used for MR experiments. One week prior to imaging, all animals were transferred from their normal rodent diet to one consisting of a folate-free rodent diet.

In vivo MR imaging. All MR experiments were performed on a Siemens Vision-Plus 1.5T MR scanner. A head radio frequency coil was used for all studies. Ten pulmonary tumor animal models (hFR-positive) were divided into two groups: control group ( $\mathrm{n}=5$, Gd-DTPA, $0.2 \mathrm{mmol} / \mathrm{Gd}^{3+} / \mathrm{kg}$ ) and experimental group ( $\mathrm{n}=5$, folate-PL-Gd-DTPA, $0.2 \mathrm{mmol} / \mathrm{Gd}^{3+} / \mathrm{kg}$ ), randomly. Folate-PL-Gd-DTPA or Gd-DTPA was injected into the caudal vein of the mice in the study using a 27-gauge catheter (Baxter Healthcare Corporation, Deerfield, IL, USA). A multi-slice spin-echo imaging sequence was used to obtain coronal (matrix $114 \times 256$; slice thickness $2 \mathrm{~mm}$ ) or transverse (matrix 114x256; slice thickness $3 \mathrm{~mm}$ ) slices under T1-weighted (TR/TE 270/14 msec). Images were acquired before contrast administration, immediately after contrast administration, and 12, 24, 48 and $72 \mathrm{~h}$ after contrast administration. The field of view was $160 \mathrm{~mm}$ for the coronal images and transverse images.

Image analysis. The coronal images were used to obtain tumor and thigh muscle signal intensity and transverse images to liver signal intensity. Of the five slices obtained for each mouse on coronal and transverse sections, respectively, the top 
A

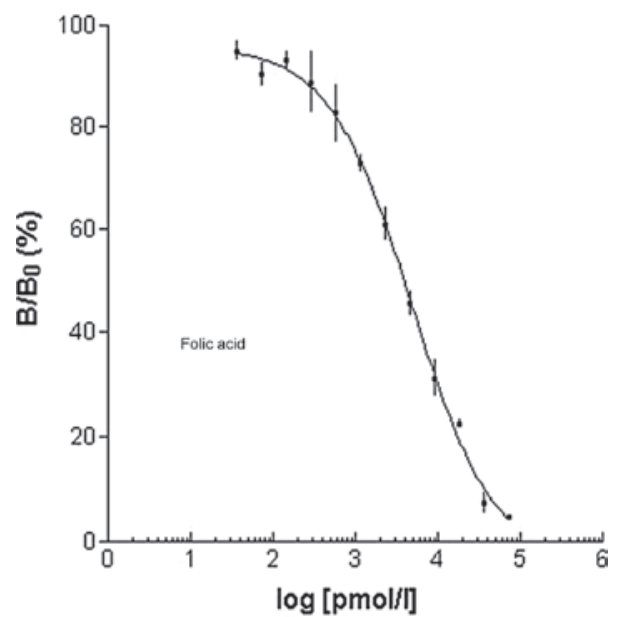

B

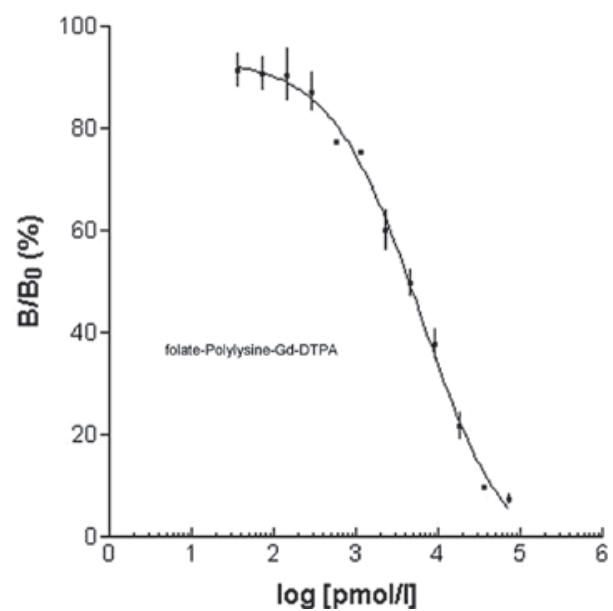

Figure 1. Log-curve between $\mathrm{B} / \mathrm{B}_{0}$ and different concentrations of $(\mathrm{A})$ folic acid or $(\mathrm{B})$ folate-PL-Gd-DTPA.

and bottom slices were not included in the data analysis to prevent confounding partial volume effects at the edges of the tumor. The signal intensities within the region of interest (i.e., tumor or reference) were obtained. The tumor, muscle and liver signal intensity was normalized to the noise standard deviation of the propotional slice reference to obtain a signal-to-noise ratio (SNR) at each of the imaging conditions (pre-injection, or different scan time-point post-injection). The percentage of contrast enhancement (PCE) was calculated according to the following: PCE $=($ SNRpost - SNRpre $) /$ SNRpre $\times 100 \%$. SNRpost was replaced with SNRpost at different scan timepoints to obtain the PCE at different scan time-points.

Statistical analysis. All data groups were analyzed and data are expressed as the means \pm standard deviation (SD). The differences in tumor, liver and muscle SNR at different times were tested using analysis of variance (ANOVA, F-test). Differences were considered significant at P-value of $<0.05$. Statistical analysis was performed using the SPSS statistical software (version 10.0; SPSS, Inc., Chicago, IL, USA).

\section{Results}

In vitro specificity studies. Prior to showing specificity of the folate-PL-Gd-DTPA binding in vivo with MRI, radioactive iodine-125, chelated to folic acid was administered to human pulmonary adenocarcinoma cells in vitro. We wanted to test whether folate-PL-Gd-DTPA was able to bind to these cells which express the folate receptor. In this competition experiment, folate-PL-Gd-DTPA exhibited a significant effect of competition inhibition to folic acid binding folate receptor. Results presented in Fig. 1 show that the folate receptorpositive cells treated with folate-PL-Gd-DTPA were similar in binding compared to folic acid.

In vivo specificity studies. Following the demonstration of the efficacy of binding of folate-PL-Gd-DTPA in vitro, we wanted to evaluate the targeting of folate-PL-Gd-DTPA to pulmonary tumor xenografts generated in vivo with MRI. Initially, we showed that folate-PL-Gd-DTPA is able to accumulate in pulmonary tumor xenografts expressing the hFR resulting in greater enhancement of the tumors compared to a non-specific

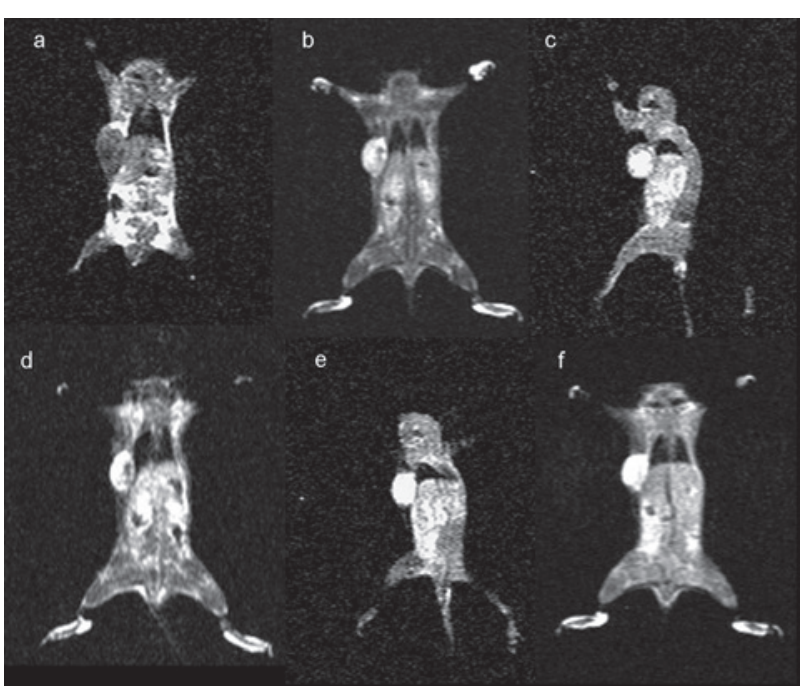

Figure 2. Coronal slices under T1-weighted tumor imaging pre- and post-injection of folate-PL-Gd-DTPA. (a) Pre-injection scanning; (b) postinjection; (c) post-12 h injection; (d) $24 \mathrm{~h}$ time-point post-injection; (e) $48 \mathrm{~h}$ time-point post-injection; (f) $72 \mathrm{~h}$ time-point post-injection.

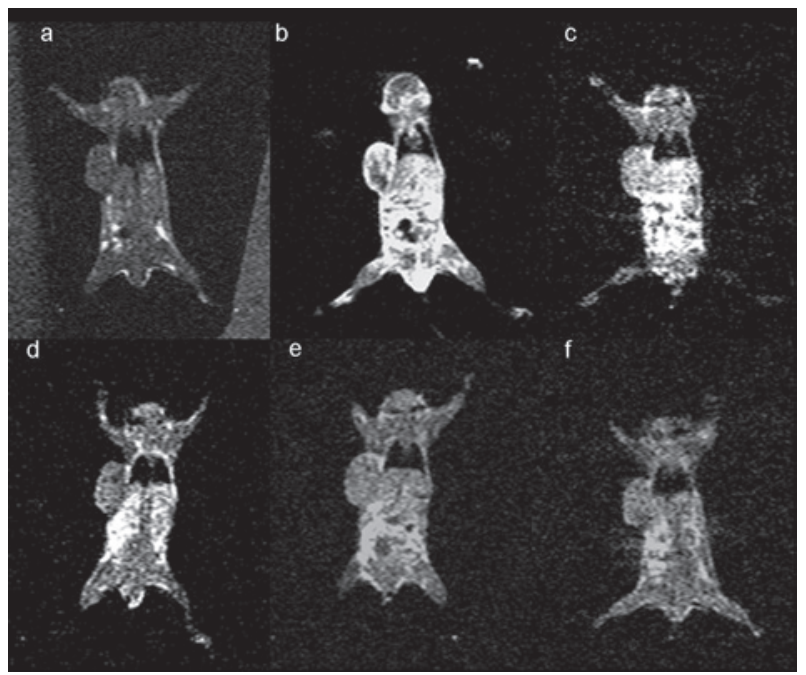

Figure 3. Coronal slices under T1-weighted tumor imaging pre- and postinjection of Gd-DTPA. (a) Pre-injection scanning; (b) post-injection; (c) post-12 h injection; (d) $24 \mathrm{~h}$ time-point post-injection; (e) $48 \mathrm{~h}$ time-point post-injection; (f) $72 \mathrm{~h}$ time-point post-injection. 
Table I. SNR of tumors pre-injection and post-injection at different scan time-points ${ }^{\mathrm{a}}$.

\begin{tabular}{lcccccc}
\hline & Pre-injection & Post-injection & $\begin{array}{c}\text { Post-12 h } \\
\text { injection }\end{array}$ & $\begin{array}{c}\text { Post-24 h } \\
\text { injection }\end{array}$ & $\begin{array}{c}\text { Post-48 h } \\
\text { injection }\end{array}$ & $\begin{array}{c}\text { Post-72 h } \\
\text { injection }\end{array}$ \\
\hline Folate-PL-Gd-DTPA (n=5) & $206.2 \pm 8.7$ & $262.8 \pm 37.1^{\mathrm{b}}$ & $305.2 \pm 20.6^{\mathrm{b}}$ & $443.8 \pm 57.2^{\mathrm{b}}$ & $464.8 \pm 31.3^{\mathrm{b}}$ & $424.1 \pm 53.4^{\mathrm{b}}$ \\
Gd-DTPA (n=5) & $174.3 \pm 6.0$ & $394.5 \pm 24.5^{\mathrm{b}}$ & $202.5 \pm 13.8$ & $190.7 \pm 16.6$ & $188.0 \pm 13.9$ & $181.4 \pm 11.9$ \\
\hline
\end{tabular}

SNR, signal-to-noise ratio. ${ }^{\text {a }} \mathrm{SNR}$ is presented as the means \pm standard deviation. ${ }^{\mathrm{b}} \mathrm{P}<0.05$.

Table II. SNR of tumors, liver and thigh muscle at pre-contrast and post-injection at different scan time-points ${ }^{\mathrm{a}}$.

\begin{tabular}{lcccccc}
\hline & Pre-injection & Post-injection & $\begin{array}{c}\text { Post-12 } \mathrm{h} \\
\text { injection }\end{array}$ & $\begin{array}{c}\text { Post-24 h } \\
\text { injection }\end{array}$ & $\begin{array}{c}\text { Post-48 } \mathrm{h} \\
\text { injection }\end{array}$ & $\begin{array}{c}\text { Post-72 } \mathrm{h} \\
\text { injection }\end{array}$ \\
\hline Tumor & $209.5 \pm 6.3$ & $268.6 \pm 39.9^{\mathrm{b}}$ & $294.9 \pm 26.8^{\mathrm{b}}$ & $453.5 \pm 20.4^{\mathrm{b}}$ & $473.4 \pm 17.0^{\mathrm{b}}$ & $445.5 \pm 51.0^{\mathrm{b}}$ \\
Liver & $240.7 \pm 20.9$ & $377.6 \pm 43.9^{\mathrm{b}}$ & $438.0 \pm 18.8^{\mathrm{b}}$ & $388.8 \pm 26.9^{\mathrm{b}}$ & $297.5 \pm 24.6^{\mathrm{b}}$ & $267.8 \pm 12.6$ \\
Muscle & $170.3 \pm 13.7$ & $208.9 \pm 37.7$ & $213.2 \pm 7.7$ & $221.7 \pm 22.6$ & $199.3 \pm 10.5$ & $232.2 \pm 35.1$ \\
\hline
\end{tabular}

SNR, signal-to-noise ratio. ${ }^{\text {a }} \mathrm{SNR}$ is presented as the means \pm standard deviation. ${ }^{\mathrm{b}} \mathrm{P}<0.05$.

agent, Gd-DTPA in the same tumor type. Forty-eight hours after the injection, a maximum $125.4 \%$ change in PCE was noted in the pulmonary tumors expressing the hFR $(n=5)$ (Fig. 2 and Table I). However, at the same time-point in the non-specific agent (Gd-DTPA) group, the change in PCE was non-significantly different from pre-injection $(\mathrm{P}>0.05)$ (Fig. 3 and Table I).

Next, we tested the specificity of folate-PL-Gd-DTPA binding to the hFR. To determine whether the PCE observed in the hFR-positive xenografts following folate-PL-Gd-DTPA administration was specific, the SNRs of tumor, liver and thigh muscle at different times were observed as control $(n=3)$. Results of the SNR of tumor, liver and thigh muscle at pre-contrast and post-injection different scan time-points are presented in Table II. Early, the tumor and liver had significant contrast enhancement after injection $(\mathrm{P}<0.05)$. The tumor contrast enhancement achieved a platform at 24-72 h after injection of folate-PL-Gd-DTPA. Meanwhile, the liver SNR decreased, and there was no difference between the values before injection and at $72 \mathrm{~h}(\mathrm{P}>0.05)$. The muscle had no contrast enhancement following different scan times after folate-PL-Gd-DTPA injection. This provided additional evidence that the contrast enhancement originally observed was most likely due to folate-PL-Gd-DTPA targeting to the tumor folate receptors.

\section{Discussion}

Currently, there is a need for contrast agents with specific localization within targeted tissues of interest in order to improve lesion to normal tissue contrast, facilitating lesion diagnosis and disease prognosis. The folate receptor is frequently overexpressed in human tumors, including pulmonary adenocarcinoma. Folic acid conjugates have been shown to bind to the folate receptor with high affinity and are taken up by tumor cells by folate receptor-mediated endocytosis. This provides a useful approach for targeted delivery of imaging or therapeutic agents into tumor cells. The aim of this study was to show specific targeting with MRI of the folate-conjugate chelate to pulmonary tumor xenografts expressing the high-affinity folate receptor.

In this study, folic acid was chosen as the ligand for the folate receptor. Compared to monoclonal antibody against the folate receptor, for example Mov18, folate conjugates are low-molecular-weight agents that have high degrees of tumor specificity, rapid systemic clearance and are potentially nonimmunogenic. Additionally, in MRI-targeted diagnosis, large amounts of the magnetic label in the tissue of interest are required in order to achieve a sufficient diagnostic signal. Unger et al (7) used a CEA antibody conjugated to approximately $1.5 \mathrm{Gd}$ atoms/antibody to enhance human colon carcinoma implanted in the thighs of hamsters. In the in vivo MRI study, there was no enhancement of the tumors due to low amounts of magnetic label in the tissue of interest. In our study, to increase the number of gadolinium per folate, polymeric gadolinium complexes were utilized, where the $\varepsilon$-amino groups of polyL-lysine were conjugated to approximately $56 \mathrm{Gd}$-DTPA molecules and then to folic acid (21).

Our competition experiment initially showed that folatePL-Gd-DTPA is able to bind to folate receptor-positive cells similar to folate binding. Next, we tested the specificity of folate-PL-Gd-DTPA binding to the hFR in vivo. The PCE increased to $125.4 \%$ following $48 \mathrm{~h}$ of folate-PL-Gd-DTPA administration. This increase was not evident in tumors treated with the non-specific agent (Gd-DTPA) at the same time-point. The latter result was expected since extracellular, fluid-space agents are usually not present $24 \mathrm{~h}$ following the injection, due to their short half-life of $20 \mathrm{~min}$ in rats and $90 \mathrm{~min}$ in humans (22). These results showed that the folate-conjugated chelates are able to accumulate in pulmonary tumors expressing the hFR. The mechanisms of enhancement may be as follows. First, the accumulation could be due to a simple blood pool effect based 
on the longer plasma half-life of the folate-conjugated chelate compared to the low-molecular weight agent. It may also be due to a passive targeting of the folate-conjugated chelate into the tumor resulting from the leakage of the agent into the tumor interstitium via hyperpermeable capillaries which are often present in tumors (23). We also compared tumor SNR with the liver and muscle SNRs following different scan times after folate-PL-Gd-DTPA injection. The results also showed that the folate-conjugated chelates are able to accumulate in pulmonary tumors expressing the hFR.

These results, however, do not differentiate between the binding of the chelate to the hFR on the cell surface, in the tumor interstitium, or both. It is possible that the enhancement may result from a combination of a prolonged interstitial residence time and specific targeting. The hFR is bound to the extracellular surface of the plasma membrane by a glycosyl-phosphatidylinositol anchor (24) and is cleaved by phospholipase $\mathrm{C}$ (25). This results in high concentrations of the soluble form of the receptor in the tumor interstitium $(26,27)$. The interstitial residence time of the targeted agent may increase with its specific binding to the soluble form of the receptor. Ideally, the results may also be the result of specific binding of the agent to the cell surface receptor, coupled with intracellular accumulation caused by receptor recycling (28) without soluble receptor binding. Finally, a combination of binding to the cell surface receptor and the soluble receptor may also occur. Regardless, the targeting is specific to the hFR and detectable by MRI.

In conclusion, we prepared macromolecular contrast agent folate-PL-Gd-DTPA, which binds to cells which express the folate receptor such as folic acid in in vitro studies. Successful MRI of folate receptor-expressing tumors in vivo demonstrate that they possess specificity targeting pulmonary tumor xenografts expressing the high-affinity folate receptor. The folate/ poly-1-lysine chelate appears highly versatile and can also be used as a common platform, by incorporating other diagnostic agents and chemical/biomolecular substances, including chemotherapeutics, in the targeted delivery approach for tumor diagnosis and therapy.

\section{Acknowledgements}

This study was supported by the National Natural Science Foundation of China (No. 30570534/C03031801), and the Science and Technology Commission of Shanghai Municipality (No. 0552nm026).

\section{References}

1. Moghimi SM and Rajabi-Siahboomi AR: Recent advances in cellular, subcellular and molecular targeting. Adv Drug Deliv Rev 41: 129-133, 2000.

2. Hood JD, Bednarski M, Frausto R, Guccione S, Reisfeld RA, Xiang R and Cheresh DA: Tumor regression by targeted gene delivery to the neovasculature. Science 296: 2404-2407, 2002.

3. Hollopeter G, Jantzen HM, Vincent D, Li G, England L, Ramakrishnan V, Yang RB, Nurden P, Nurden A, Julius D and Conley PB: Identification of the platelet ADP receptor targeted by antithrombotic drugs. Nature 409: 202-207, 2001.

4. Low RN, Saleh F, Song SYT, Shiftan TA, Barone RM, Lacey CG and Goldfarb PM: Treated ovarian cancer: comparison of MR imaging with serum CA-125 level and physical examination - a longitudinal study. Radiology 211: 519-528, 1999.
5. Ke CY, Mathias CJ and Green MA: The folate receptor as a molecular target for tumor-selective radionuclide delivery. Nucl Med Biol 30: 811-817, 2003.

6. Brasch RC: Rationale and applications for macromolecular Gd-based contrast agents. Magn Reson Med 22: 282-287, 1994.

7. Unger EC, Totty WG, Neufeld DM, Otsuka FL, Murphy WA, Welch MS, Connett JM and Philpott GW: Magnetic resonance imaging using gadolinium labeled monoclonal antibody. Invest Radiol 20: 693-700, 1985

8. Gohr-Rosenthal S, Schmit-Willich H, Ebert W and Conrad J: The demonstration of human tumors on nude mice using gadoliniumlabeled monoclonal antibodies for magnetic resonance imaging. Invest Radiol 28: 789-795, 1993.

9. Zuckier LS, Rodriguez LB and Scharff MD: Immunologic and pharmacologic concepts of monoclonal antibodies. Semin Nucl Med 28: 166-186, 1989.

10. Leamon CP and Low PS: Delivery of macromolecules into living cells: a method that exploits folate receptor endocytosis. Proc Natl Acad Sci USA 88: 5572-5576, 1991.

11. Leamon CP and Low PS: Cytotoxicity of mormordin-folate conjugates in cultured human cells. J Biol Chem 267: 24966-24971, 1992.

12. Leamon CP, Pastan I and Low PS: Cytotoxicity of folate-pseudomonas exotoxin conjugates toward tumor cells. Contribution of translocation domain. J Biol Chem 268: 24847-24854, 1993.

13. Antony AC: Folate receptors. Annu Rev Nutr 16: 501-521, 1996.

14. Garin-Chesa P, Campbell I, Saigo PE, Lewis JL Jr, Old LJ and Rettig WJ: Trophoblast and ovarian cancer antigen LK26. Sensitivity and specificity in immunopathology and molecular identification as a folate-binding protein. Am J Pathol 142: 557-567, 1993.

15. Ross JF, Chaudhuri PK and Ratnam M: Differential regulation of folate receptor isoforms in normal and malignant tissues in vivo and in established cell lines. Physiologic and clinical implications. Cancer 73: 2432-2443, 1994.

16. Lu Y and Low PS: Folate-mediated delivery of macromolecular anticancer therapeutic agents. Adv Drug Deliv Rev 54: 675-693, 2002.

17. Sudimack $\mathrm{J}$ and Lee RJ: Targeted drug delivery via the folate receptor. Adv Drug Deliv Rev 41: 147-162, 2000.

18. Xu L, Pirollo KF and Chang EH: Tumor-targeted p53-gene therapy enhances the efficacy of conventional chemo/radiotherapy. J Control Release 74: 115-128, 2001.

19. Maziarz KM, Monaco HL, Shen F and Ratnam M: Complete mapping of divergent amino acids responsible for differential ligand binding of folate receptors alpha and beta. J Biol Chem 274: 11086-11091, 1999.

20. Shen F, Ross JF, Wang X and Ratnam M: Identification of a novel folate receptor, a truncated receptor, and receptor type in hematopoietic cells: cDNA cloning, expression, immunoreactivity, and tissue specificity. Biochem 33: 1209-1215, 1994.

21. Yuan Z, Liu SY, Xiao XS, Zhong GR and Jiang QJ: Folate-polyL-lysine-Gd-DTPA as MR contrast agent for tumor imaging via folate receptor-targeted delivery. Zhonghua Yi Xue Za Zhi 87: 673-678, 2007 (In Chinese).

22. Brasch RC: Rationale and applications for macromolecular Gd-based contrast agents. Magn Reson Med 22: 282-287, 1991.

23. Jain RK: Transport of molecules in the tumor interstitium: a review. Cancer Res 47: 3039-3051, 1987.

24. Rijnboutt S, Jansen G, Posthuma G, Hynes JB, Schornagel JH and Strous GJ: Endocytosis of GPI-linked membrane folatereceptor-[alpha]. J Cell Biol 132: 35-47, 1996.

25. Luhrs CA and Slomiany BL: A human membrane-associated folate binding protein is anchored by a glycosyl-phospatidylinositol tail. J Biol Chem 264: 21446-21449, 1989.

26. Kane MA, Elwood PC, Portilla RM, Antony AC and Kolhouse JF: The interrelationship of the soluble and membrane-associated folate-binding proteins in human KB cells. J Biol Chem 261: $15625-15631,1986$.

27. Antony AC: The biological chemistry of folate receptors. Blood 79: 2807-2820, 1992.

28. Wiener EC, Konda S, Shadron A, Brechbiel M and Gansow O: Targeting dendrimer-chelates to tumors and tumor cells expressing the high-affinity folate receptor. Invest Radiol 32: 748-754, 1997. 